\title{
Residual Stress Analysis of Ceramic Thermal Barrier Coating Based on Thermal Spray Process"
}

\author{
Masayuki ARAI $^{* *}$, Eiji WADA ${ }^{* * *}$ and Kikuo KISHIMOTO ${ }^{* * * *}$ \\ **Materials Science Research Lab., Central Research Institute of Electric Power Industry, \\ 2-11-1 Iwado-kita, Komae-shi, Tokyo 201-8511, Japan \\ E-mail: marai@criepi.denken.or.jp \\ ***Department of Mechanical Engineering and Science, Tokyo Institute of Technology, \\ 2-12-1, O-okayama, Meguroku, Tokyo 152-8552, Japan \\ ${ }^{* * * *}$ Department of Mechanical Engineering and Science, Tokyo Institute of Technology, \\ 2-12-1, O-okayama, Meguro-ku, Tokyo 152-8552, Japan \\ E-mail: kkishimo@mep.titech.ac.jp
}

\begin{abstract}
Residual stress is generated in ceramic thermal barrier coatings (TBCs), which were sprayed by a plasma spray technology, due to the difference in coefficients of thermal expansion between the coating and the substrate. Previous experimental results obtained by the X-ray diffraction method indicated that the residual stress at the ceramic coating surface is tensile and could lead to TBC failure such as cracking and spalling of the ceramic coating. In this study, a numerical model that can predict the residual stress exactly is proposed by taking into account a thermal spray process. This numerical model is a layer-buildup model based on a shear-lag theory, and the residual stress contribution comes from two kinds of the following stress components: (1) quenching stress, which was generated in molten spray particles impinged onto the substrate, and (2) thermal stress, which was generated due to differences in thermal expansion between the deposited particle and the underlying substrate. It is shown herein that residual stress predicted by the proposed numerical model coincided with the experimental one obtained by the strain gage technique, with a good level of accuracy.
\end{abstract}

Key words: Ceramic Thermal Barrier Coating, Thermal Spray Deposition, Plasma Spraying and Residual Stress

\section{Introduction}

Ceramic thermal barrier coatings (TBCs), which play an important role in insulating hot components such as blades of gas turbines from high heat flux and/or high-temperature environments, are composed of partially stabilized zirconia $\left(8 \mathrm{wt} \% \mathrm{Y}_{2} \mathrm{O}_{3}-\mathrm{ZrO}_{2}\right)$ and metallic bonds (MCrAlY; $\mathrm{M}$ is $\mathrm{Ni}$ and/or $\mathrm{Co}$ ) and are generally deposited using plasma-spraying technology. Residual stress in TBCs, which is generated during the spraying process and service, is a considerable mechanical factor in the performance and lifetime of the coated components. The residual stress could lead to cracking within the ceramic coating by subjecting it to thermal shock $^{(1)}$, and to spallation of ceramic coating brought about by interfacial crack propagation ${ }^{(2)}$. Thus, it is necessary to grasp comprehensively the residual stress in TBCs sprayed using a plasma spraying process, in order to assess precisely the lifetime up to the occurrence of TBC failure.

\footnotetext{
${ }^{*}$ Received 13 June, 2007 (No. T1-05-1086) Japanese Original : Trans. Jpn. Soc. Mech. Eng., Vol. 72, No. 717, A (2006), pp.676-682 (Received 12 Oct., 2005) [DOI: 10.1299/jmmp.1.1251]
} 


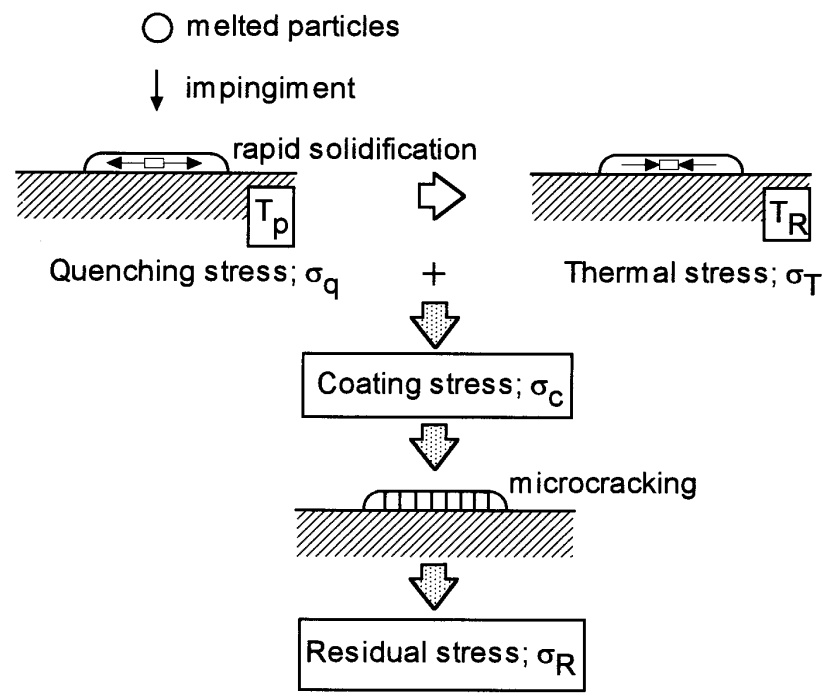

Fig. 1 Schematic illustration of impact, spreading and solidification of a single splat

A plasma spraying process is characterized by the impingement of molten particles onto a substrate and rapid solidification of the particles spread along the substrate ${ }^{(3)}$. The single-particle deposition process is shown schematically in Fig.1. Spherical-shaped molten particles are impacted into a high-temperature plasma flow and spread on the surface of substrate. Shrinkage of the flattened particle (splat) is constrained by the underlying substrate, and a tensile microstress (quenching stress) is generated in the splat during cooling up to the point at which it reaches the pre-heated substrate temperature $\left(T_{p}\right)$. The quenching stress could bring about cracking normal to the substrate surface and originate a lamella structure known as a typical microstructure in TBCs. As the other stress source, thermal stress occurs during cooling from the pre-heated substrate temperature to room temperature $\left(T_{R}\right)$ after finishing a complete particle flattening and solidification process. Due to differences in thermal expansion between the deposited particle and the substrate, thermal stress develops in the dual-layered structure. This continuous deposition process affects the residual stress value in TBCs, and consequently it needs to be considered in a model for predicting the residual stress.

Much effort has been made, from both experimental and analytical approaches, to estimate the residual stress generated in TBCs. Teixeira et al. ${ }^{(4)}$ have conducted residual stress measurements for material combination $8 \mathrm{wt} \% \mathrm{Y}_{2} \mathrm{O}_{3}-\mathrm{ZrO}_{2} / \mathrm{NiCoCrAlY} /$ Inconel 617 by $\mathrm{X}$-ray diffraction using a $\sin ^{2} \Psi$ technique. They also have considered the residual stress model based on classical beam theory, in which homogeneous materials are completely bonded at the interface and thermal stress alone is assumed to be dominant in residual stress formation. Comparison between X-ray results and those predicted by the model has revealed that residual stress predicted by the model is higher than that measured experimentally. This prediction error was caused by quenching stress not taken into account in the analysis model. $\mathrm{Ng}$ et al ${ }^{(5)}$ have developed a finite element analysis model to predict residual stress in TBCs. The continuous deposition process was idealized and the assumption of a multiple layer-by-layer coating structure was incorporated into the analysis model. In numerical calculations, comprehensive heat transfer analysis was conducted using time-temperature history measured during the plasma spraying process. However, the numerical results also showed much higher residual stress in comparison with that obtained by the curvature measurement method. In both models, it should be noted that microstress, microcracking and splat boundary formation generated in the splat during the deposition process is not sufficiently taken into consideration in the models, in spite of a significant 


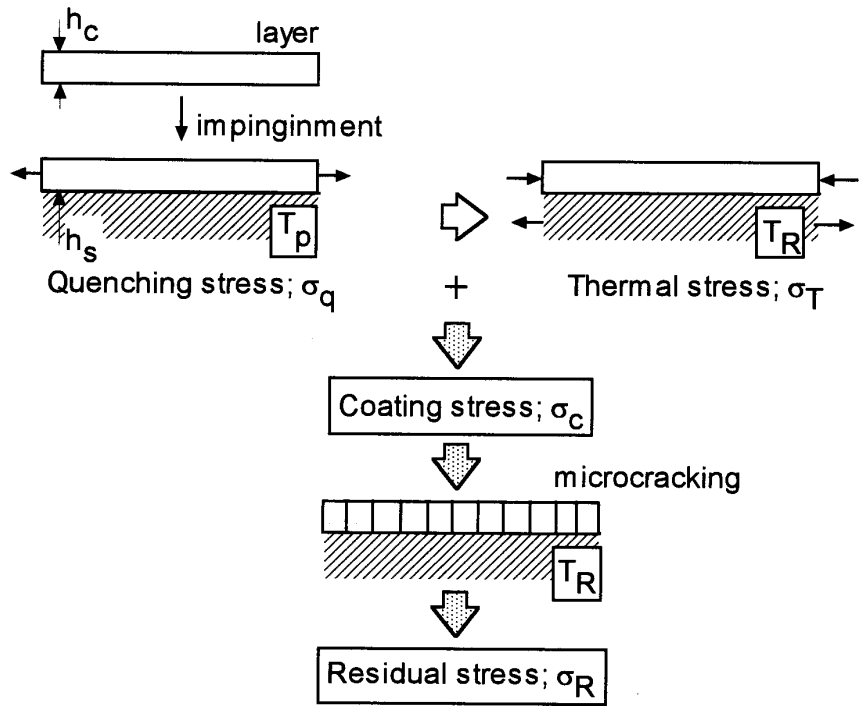

Fig. 2 Layer-buildup model by deposition layer impinging directly onto substrate

factor for a residual stress generation mechanism.

In this study, we propose a comprehensive numerical model to estimate residual stress in TBCs generated during the plasma spraying process, based on the assumption of layerbuildup structure modeled as the continuous splat deposition process. The model is developed upon a shear lag theory for taking into account sliding deformation at splat boundaries and stress relaxation caused by cracking in splat. The strain gage method, which is a measurement technique based on residual strain obtained by releasing the ceramic coating from metallic bond coating and substrate, is employed to measure the residual stress. We compare the results predicted by our numerical model with experimental results in order to verify the proposed model. In addition, best control parameters in the plasma spraying process to reduce residual stress are examined by conducting numerical analysis.

\section{Residual Stress Analysis}

\subsection{Layer-Buildup Model}

In this study, a continuous molten particle deposition process is idealized by impingement of a single layer (called the "deposition layer".) onto a substrate. This idealized model representation is shown in Fig. 2. Residual stress is composed of two kinds of the following contributions: quenching stress, which is caused by contraction of the deposition layer on the underlying substrate, and thermal stress, which is generated by a thermal expansion mismatch between the deposition layer and the substrate.

The deposition layer exposed into a high-temperature plasma flow with spray temperature $T_{m}$ impinges onto the substrate with pre-heated temperature $T_{p}$ through the drifting force of the plasma gas flow. The deposition layer is in melting/liquid state if the temperature in the deposition layer is also the same as the spray temperature. This deposition layer deposits and spreads on the surface of the substrate when impacting onto the substrate. The temperature in the deposition layer is cooled quickly up to the point of reaching the pre-heated substrate temperature, and a contraction of the deposition layer is generated on the underlying substrate. This contraction brings about quenching stress in the deposition layer as well as single splat contraction as shown in Fig. 1. The quenching stress will be developed by a temperature drop from the solidification temperature $T_{\mathrm{s}}$ to the pre-heated substrate temperature $T_{p}$, because the effective temperature for causing thermal stress is limited in the region lower than the solidification temperature. The quenching stress 


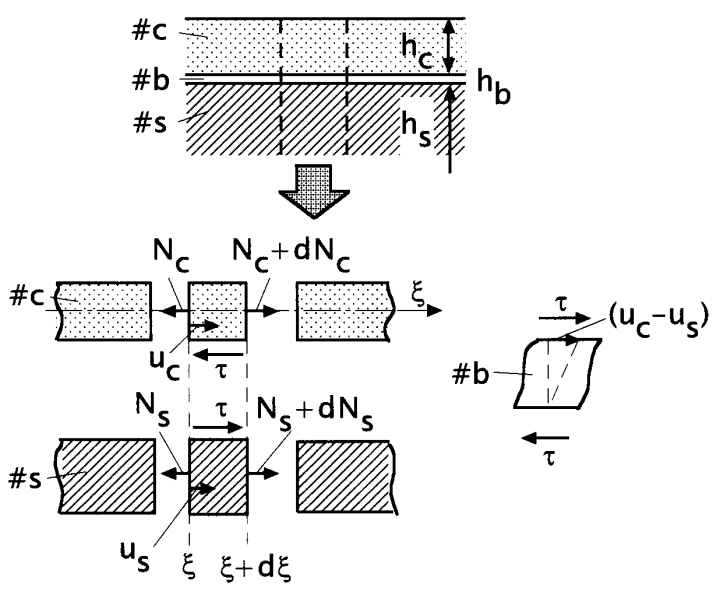

Fig. 3 Deposition layer used in this study

generated during cooling will also cause cracking normal to the substrate surface as well as cracking phenomena observed in a single splat. After the temperature in the deposition layer reaches the pre-heated substrate temperature, thermal stress arises in an overall dual-layered structure attached the deposition layer with a temperature drop from the pre-heated substrate temperature to room temperature. Consequently, residual stress in TBC can be derived by summing the quenching stress and the thermal stress.

\subsection{Formulation using Shear-Lag Theory}

The model utilized for formulation of coating stresses generated in the deposition layer during the plasma spraying process is shown in Fig. 3. Here, it should be noted that coating stress includes both quenching stress and thermal stress. In this study, a shear-lag theory is applied to obtain the coating stress, taking into account the fact that stress relaxation occurred in the deposition layer due to cracking.

The deposition layer with thickness $h_{c}$ (shown as \#c in Fig. 3 ) is bonded to the substrate with thickness $h_{s}$ (shown as \#s in Fig. 3), assuming a binding layer with thickness $h_{b}$. All dimensions have unit width.

The neutral axis is taken at the center in the deposition layer in Fig. 3. The one-dimensional coordinate $\xi$ is taken along this neutral axis. Equilibrium equation for resultant force along the $\xi$ axis in the deposition layer gives following differential equation:

$$
\frac{d N_{c}}{d \xi}=\tau
$$

where $\mathrm{N}_{\mathrm{c}}$ is axial force along the neutral axis in the deposition layer and $\tau$ is shear stress at the bottom of the deposition layer. The equilibrium equation for the substrate also gives:

$$
\frac{d N_{s}}{d \xi}=-\tau
$$

where $\mathrm{N}_{\mathrm{s}}$ is axial force in the substrate.

Assuming that deformation in the deposition layer/substrate boundary progresses elastically with sliding, the constitutive equation for the binding layer can be expressed by:

$$
\tau=\mu_{b} \frac{u_{c}-u_{s}}{h_{b}}
$$


where $\mu_{\mathrm{b}}$ is shear modulus of the binding layer, $\mathrm{u}$ is sliding displacement and subscripts $\mathrm{c}$ and $\mathrm{s}$ indicate the deposition layer and the substrate, respectively.

The strains under plane stress in the deposition layer and the substrate are obtained by summing mechanical and thermal strains as follows:

$$
\begin{aligned}
& \frac{d u_{c}}{d \xi}=\frac{N_{c}}{E_{c} h_{c}}+\int_{T_{k}}^{T} \alpha_{c}(T) d T \\
& \frac{d u_{s}}{d \xi}=\frac{N_{s}}{E_{s} h_{s}}+\int_{T_{k}}^{T} \alpha_{s}(T) d T
\end{aligned}
$$

where $\mathrm{E}$ is elastic modulus, $\alpha$ is the coefficient of thermal expansion (CTE), $\mathrm{T}$ is temperature, and $T_{k}$ takes $T_{s}$ when calculating quenching stress or $T_{p}$ when calculating thermal stress in numerical procedure. It should be noted that the elastic modulus means in-plane elastic modulus in a ceramic coating. If we consider the issue of plane strain, $\mathrm{E} /\left(1-v^{2}\right)$ is placed into $\mathrm{E}$ and $(1+v) \alpha$ is placed into $\alpha$.

Differentiating Eq. (4) and substituting Eq. (1) into Eq. (2), the following differential equations can be obtained.

$$
\begin{aligned}
& \frac{d^{2} u_{c}}{d \xi^{2}}=\frac{1}{E_{c} h_{c}} \frac{d N_{c}}{d \xi}=+\frac{1}{E_{c} h_{c}} \tau \\
& \frac{d^{2} u_{s}}{d \xi^{2}}=\frac{1}{E_{s} h_{s}} \frac{d N_{s}}{d \xi}=-\frac{1}{E_{s} h_{s}} \tau
\end{aligned}
$$

Taking the difference of Eqs. (5),

$$
\frac{d^{2}\left(u_{c}-u_{s}\right)}{d \xi^{2}}=\left(\frac{1}{E_{c} h_{c}}+\frac{1}{E_{s} h_{s}}\right) \tau
$$

Substituting Eq. (3) into Eq. (6), the following differential equation related to shear stress can be obtained.

$$
\frac{d^{2} \tau}{d \xi^{2}}=\frac{\mu_{b}}{h_{b}}\left(\frac{1}{E_{c} h_{c}}+\frac{1}{E_{s} h_{s}}\right) \tau
$$

The general solution for this differential equation is given by,

$$
\tau=A \exp (\beta \xi)+B \exp (-\beta \xi)
$$

where

$$
\beta^{2}=\frac{\mu_{b}}{h_{b}}\left(\frac{1}{E_{c} h_{c}}+\frac{1}{E_{s} h_{s}}\right)
$$

Substituting Eq. (8) into Eq. (1) and (2) and integrating it,

$$
\begin{aligned}
& N_{c}=\frac{A}{\beta} \exp (\beta \xi)-\frac{B}{\beta} \exp (-\beta \xi)+C \\
& N_{s}=-N_{c}
\end{aligned}
$$

where constants $\mathrm{A}$ and $\mathrm{B}$ can be determined from the boundary condition. Constant $\mathrm{C}$ can 
be obtained from Eq. (3), Eq. (4), Eq. (9) and Eq. (10) as follows:

$$
C=\frac{E_{c} E_{s} h_{c} h_{s}}{E_{c} h_{c}+E_{s} h_{s}} \int_{T_{k}}^{T}\left(\alpha_{s}-\alpha_{c}\right) d T
$$

By assuming that axial forces at the edges $(\xi=0$ and $l)$ of deposition layer are zero, we have $\mathrm{N}_{\mathrm{c}}(0)=0$ and $\mathrm{N}_{\mathrm{c}}(l)=0$ as the boundary condition. This boundary condition determines both constants A and B. Consequently, the coating stress generated in the deposition layer is expressed:

$$
\sigma_{c}=\frac{N_{c}}{h_{c}}=\frac{\sinh \beta(\xi-l)-\sinh \beta \xi+\sinh \beta l}{\sinh \beta l} \frac{E_{c} E_{s} h_{s}}{E_{c} h_{c}+E_{s} h_{s}} \int_{T_{k}}^{T}\left(\alpha_{s}-\alpha_{c}\right) d T
$$

\section{Residual Stress Analysis}

\subsection{Material Preparation and Thermal Spraying Conditions}

The thermal spraying powders employed in this study are $8 \mathrm{wt} \% \mathrm{Y}_{2} \mathrm{O}_{3}-\mathrm{ZrO}_{2}$ (METCO 204NS supplied by Sultzer Metco) and CoNiCrAlY (AMDRY 9954 supplied by Sultzer Metco). Scanning electron microscope observation of ceramic coating powders is shown in Fig. 4. The powder utilized has an almost spherical shape. Ni-based superalloy (IN738LC) plates with geometry of width $12.5 \mathrm{~mm}$, length $40 \mathrm{~mm}$ and thickness $\mathrm{h}_{\mathrm{s}}=1.0,2.0,3.0$ and $4.0 \mathrm{~mm}$ were prepared as a substrate. These Ni-based superalloy plates were grit-blasted prior to the plasma spraying process. After ultrasonic cleaning in acetone, CoNiCrAlY and then ceramic powders were sprayed on the substrate surface by using a plasma torch (M-1000+F4) and a gas mixture of Argon and $\mathrm{H}_{2}$. Ceramic coating was deposited up to a thickness of $0.5 \mathrm{~mm}$, and CoNiCrAlY was deposited up to a thickness of $0.1 \mathrm{~mm}$. In this study, a thermocouple was attached to the rear side of the plate-shape sample in order to obtain the time-temperature history during the plasma-spraying process.

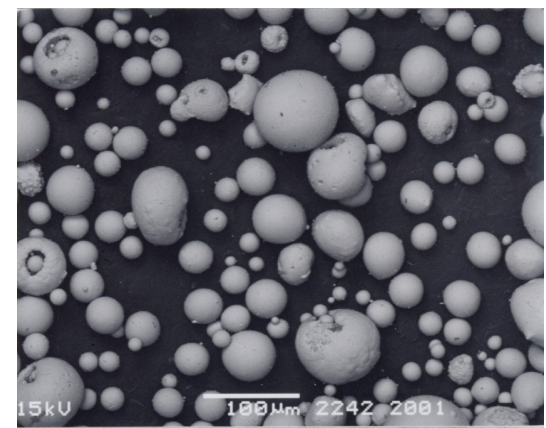

Fig. 4 Ceramic thermal spray particles used in this study

\subsection{Numerical Analysis Procedure}

This section gives an explanation of the numerical analysis procedure to predict residual stress based on layer-buildup model.

Quenching stress analysis procedure:

(Step 1) Powder materials, solidification temperature and pre-heated substrate temperature are set prior to the numerical analysis.

(Step 2) Deposition layer length is set to $l$ and coating stress is set to zero. Initial 
temperature in the deposition layer is set to the solidification temperature $T_{\mathrm{s}}$.

(Step 3) Incremental coating stress $\Delta \sigma_{\mathrm{c}}$ for associated temperature change $\Delta \mathrm{T}$ is calculated using the following equation:

$$
\Delta \sigma_{c}=\frac{\sinh \beta\left(\xi-\frac{l}{2^{n-1}}\right)-\sinh \beta \xi+\sinh \beta \frac{l}{2^{n-1}}}{\sinh \beta \frac{l}{2^{n-1}}} \frac{E_{c} E_{s} h_{s}}{E_{c} h_{c}+E_{s} h_{s}}\left(-\alpha_{c}\left(T_{i}\right)\right) \Delta T
$$

where $\mathrm{i}$ indicates the number of incremental calculations $(\mathrm{i}=1,2, \ldots$.$) and \mathrm{T}_{\mathrm{i}}=\mathrm{T}_{\mathrm{i}-1}+\Delta \mathrm{T}$. The term $\left(\frac{l}{2^{n-1}}\right)$ means length divided by cracking generated in the deposition layer.

(Step 4) The coating stress at step i can be obtained from $\sigma_{c}{ }^{i}=\sigma_{c}{ }^{i}-1+\Delta \sigma$. If this coating stress does not reach the fracture strength $\sigma_{f}$ of the freestanding ceramic coating or the temperature $T_{i}$ is lower than the pre-heated substrate temperature $T_{p}$, the incremental calculation again returns to step (3).

(Step 5) If the coating stress exceeds the fracture strength, step $n$ is progressed to $n+1$ by cracking initiation in the deposition layer. The coating stress at step $n+1$ is calculated using:

$$
\sigma_{c}^{i}=\frac{\sinh \beta\left(\xi-\frac{l}{2^{n-1}}\right)-\sinh \beta \xi+\sinh \beta \frac{l}{2^{n-1}}}{\sinh \beta \frac{l}{2^{n-1}}} \frac{E_{c} E_{s} h_{s}}{E_{c} h_{c}+E_{s} h_{s}} \int_{T_{s}}^{T_{i}}\left(-\alpha_{c}\right) d T
$$

and then the subsequent calculation returns to step (3) with Eq. (14) as an initial coating stress.

Thermal stress analysis procedure:

(Step 6) The incremental coating stress $\Delta \sigma_{\mathrm{c}}$ for the associated incremental temperature $\Delta \mathrm{T}$ can be obtained by,

$$
\Delta \sigma_{c}=\frac{\sinh \beta\left(\xi-\frac{l}{2^{N-1}}\right)-\sinh \beta \xi+\sinh \beta \frac{l}{2^{N-1}}}{\sinh \beta \frac{l}{2^{N-1}}} \frac{E_{c} E_{s} h_{s}}{E_{c} h_{c}+E_{s} h_{s}}\left(\alpha_{s}\left(T_{i}\right)-\alpha_{c}\left(T_{i}\right)\right) \Delta T
$$

where N-1 is final number of incremental calculations after the quenching stress analysis procedure.

(Step 7) The coating stress at step $i$ is obtained from $\sigma_{c}{ }^{i}=\sigma_{c}{ }^{i-1}+\Delta \sigma$. If the temperature $T_{i}$ is higher than room temperature $T_{r}$, the calculation returns to step (6). If the temperature $T_{i}$ reaches room temperature, all calculations are finished. The coating stress at the final step consequently gives the residual stress in TBC.

\subsection{Measurement Procedure of Strain Gage Method}

Strain gage method ${ }^{(6)}$ was employed to measure residual stress generated in the ceramic coating. Strain gage was attached on the surface of the ceramic TBC coated specimen. The strain gage and lead wire were covered with a protector for preventing chemical damage. An electrolytic polishing process was performed for dissolving the substrate and CoNiCrAlY. Strain was monitored continuously through the electrolytic polishing process and finally residual strain after dissolving CoNiCrAlY and substrate was obtained as shown in Fig.5, for instance. The residual stress $\sigma_{R}$ at the surface of the ceramic TBC coated 
Table 1 Analysis parameters used in this study

\begin{tabular}{|c|c|c|}
\hline \multicolumn{2}{|c|}{ Initial coating length I[mm] } & 12.5 \\
\hline \multirow{3}{*}{ Young's modulus [GPa] } & Coating & 200 \\
\hline & Binding layer & 50 \\
\hline & IN738LC & 207 \\
\hline \multicolumn{2}{|c|}{ Binding layer thickness $h_{b}$} & $h_{c} / 5$ \\
\hline \multirow{2}{*}{ CTE $X 10^{-6}[1 / K]$} & Coating & $\begin{array}{l}-49.933+0.68174 \mathrm{~T}-3.2474 \mathrm{~T}^{2}+8.2813 \times 10^{-6} \mathrm{~T}^{3}(\mathrm{~T}<900 \mathrm{~K}) \\
9.9694(\mathrm{~T}>900 \mathrm{~K})\end{array}$ \\
\hline & IN738LC & $\begin{array}{l}8.566+1.0463 \times 10^{-2} \mathrm{~T}+9.0062 \times 10^{-7} \mathrm{~T}^{2}-7.1982 \times 10^{-10} \mathrm{~T}^{3}(\mathrm{~T}<900 \mathrm{~K}) \\
18.187(\mathrm{~T}>900 \mathrm{~K})\end{array}$ \\
\hline \multicolumn{2}{|c|}{ Fracture stress $[\mathrm{MPa}]$} & $\begin{array}{l}1265.4-1.9759 \mathrm{~T}+1.0405 \times 10^{-3} \mathrm{~T}^{2}-1.7265 \times 10^{-7} \mathrm{~T}^{3}(\mathrm{~T}<1500 \mathrm{~K}) \\
103.13-0.049474 \mathrm{~T}+1.6412 \times 10^{-5} \mathrm{~T}^{2}-1.8276 \times 10^{-9} \mathrm{~T}^{3}(1500 \mathrm{~K}<\mathrm{T}>3000 \mathrm{~K})\end{array}$ \\
\hline
\end{tabular}

CTE; Coefficient of thermal expansion

specimen was obtained using the product of the residual strain $\varepsilon_{\mathrm{R}}$ and elastic modulus $\mathrm{E}$ as follows:

$$
\sigma_{R}=-E \varepsilon_{R}
$$

\subsection{Results and Discussion}

Table 1 shows physical properties used for this numerical analysis. The physical properties involve elastic modulus, CTE for deposition layer and substrate, and fracture strength of the freestanding ceramic coating, which are dependent on an ambient temperature. The elastic modulus and fracture strength of the freestanding ceramic coating were attributed to those of the ceramic powder itself ${ }^{(7)}$. Generally speaking, it was known that the elastic modulus of the bulk ceramic coating has approximately ten times the overall elastic modulus of the ceramic coating layer, because the microstructure of the ceramic coating includes a lamellar structure, microcracks, and an open pore ${ }^{(8)}$. The benefit for our proposed analysis method is addressed in considering those microstructure influences in a layer-buildup model based on a shear-lag theory. In this numerical analysis, solidification temperature $T_{s}$ was set to $2000 \mathrm{~K}$ and pre-heated substrate temperature $T_{p}$ was $523 \mathrm{~K}$, both of which were obtained from continuous monitoring of temperature at the rear side of the coated sample during the plasma spraying process. Poisson's ratio $v_{c}$ of deposition layer was 0.07 , and $v_{\mathrm{s}}$ for the substrate was 0.3 . The residual stress analysis was conducted under a plane strain.

Figure 6 shows variation of coating stress with temperature in the deposition layer, and

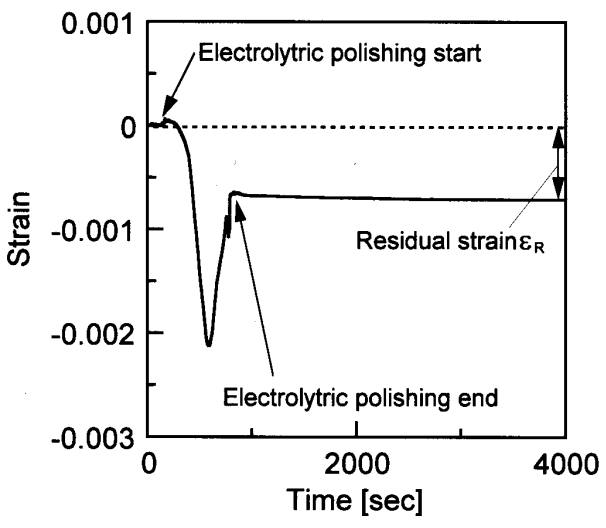

Fig. 5 Variation of strain with time during electrolytic polishing process 


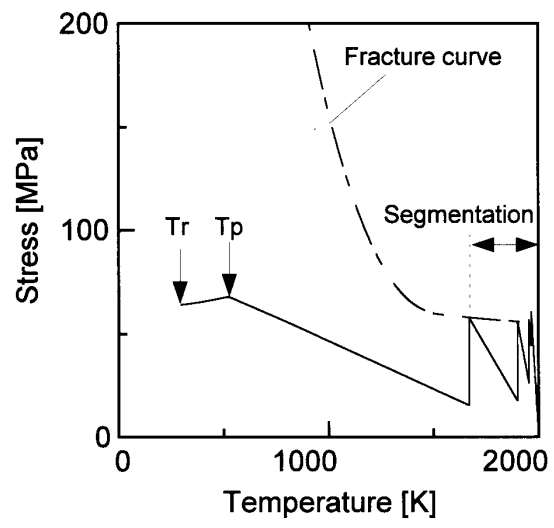

Fig. 6 Variation of coating stress with temperature during thermal spray process

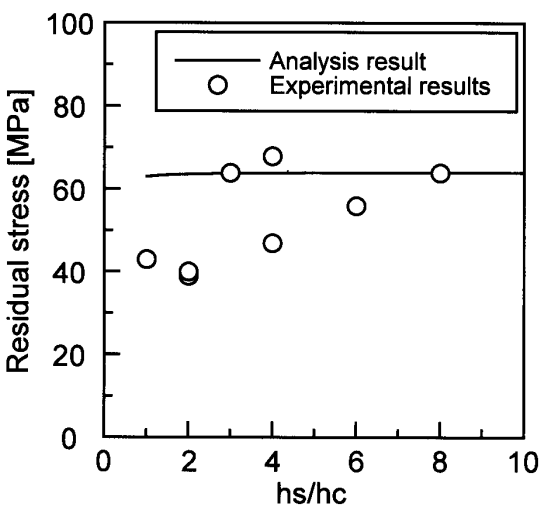

Fig. 7 Comparison between residual stress and thickness ratio of substrate to ceramic coating

also includes fracture strength curve of the ceramic, shown as a broken line. This analysis result was obtained by assuming that the deposition layer thickness $h_{c}$ is $0.5 \mathrm{~mm}$, substrate thickness $h_{s}$ is $4.0 \mathrm{~mm}$ and pre-heated substrate temperature $T_{p}$ is $523 \mathrm{~K}$. The coating stress (that is, quenching stress) increases as temperature decreases from the solidification temperature, and then the coating stress is dropped by means of cracking occurring in the deposition layer. Successive analysis shows coating stress increasing with decreasing temperature. With temperature decreasing up to the point of reaching the pre-heated substrate temperature, the coating stress increases and decreases cyclically, generating numerous cracks in the deposition layer. The coating stress increases with a lower slope. This lower slope arises due to the overall elastic modulus of the deposition layer having been significantly reduced by the occurrence of numerous cracks in the deposition layer. By conducting further analysis after reaching the pre-heated substrate temperature, tensile residual stress is finally generated at room temperature as shown in the figure. This trend as seen in the coating stress-temperature history is reasonable in comparison with the reference (9) related to coating stress measured previously by the curvature technique.

Fig. 7 shows a comparison between experimental results by the strain gage method and results predicted by the layer-buildup model. The predicted residual stress is in good agreement with the experimental results in higher substrate thickness especially. The measurement results increase with the substrate thickness and finally approach $60 \mathrm{MPa}$ in the thicker region. However, somewhat of a difference between experimental and analytical results was observed in the thinner thickness region. This difference was considered to have been caused by ignoring the equilibrium equation of bending moment in the layer-buildup model; that is, by assuming a constant thermal stress distribution in the dual-layered 


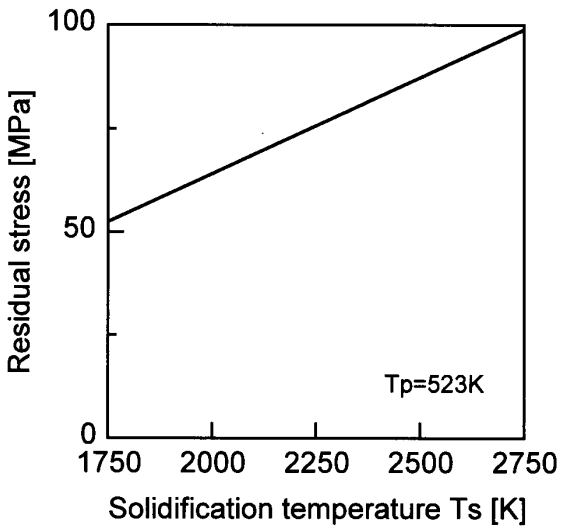

Fig. 8 Variation of residual stress with solidification temperature of thermal spray particle

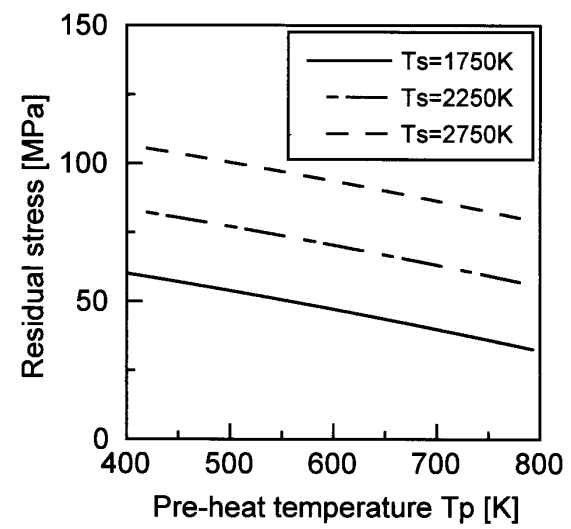

Fig. 9 Variation of residual stress with pre-heated substrate temperature

structure. An improvement for prediction accuracy in a wide range of coating thicknesses will be made by taking this bending-moment effect into account in the model.

The influence of particle materials and spraying conditions on residual stress was examined successively. In this study, solidification temperature and pre-heated substrate temperature under those conditions were attended to through parametric analysis. Figure 8 shows the results of simulating residual stress when changing solidification temperature under $h_{c}=0.5 \mathrm{~mm}, h_{s}=4.0 \mathrm{~mm}$ and $T_{p}=523 \mathrm{~K}$. The results reveal that the residual stress increases with the solidification temperature of spray powder.

Figure 9 shows the influence of pre-heated substrate temperature on the residual stress obtained under $h_{c}=0.5 \mathrm{~mm}$ and $h_{s}=4.0 \mathrm{~mm}$ as well as the results in Fig. 8. This graph includes three kinds of solidification temperatures: $T_{\mathrm{s}}=1750 \mathrm{~K}, 2250$ and $2750 \mathrm{~K}$. Residual stress decreases with increasing pre-heated substrate temperature, and also residual stress increases with solidification temperature. This result recommends that residual stress in TBC sprayed by plasma spraying technique could be reduced with higher pre-heated substrate temperature conditions and/or lower solidification temperature as sprayed powder material. However, it is also noted that optimum spraying conditions should be found between spraying parameters for reducing mechanically residual stress and a high-temperature oxidation limitation that could be progressed in the powder surface into a plasma heat flow.

\section{Conclusion}

A numerical model predicting residual stress in TBC sprayed by a plasma spraying 
technique was proposed. The model is one based on an impingement of a deposition layer on the substrate as a modeling of a continuous deposition process for numerous particles, and a shear-lag theory was applied to express stress relaxation caused by cracking in ceramic coating. In order to validate the proposed model, residual stress was measured by a strain gage method, releasing constraint strain from the substrate. It was shown that the results predicted by the numerical analysis were in good agreement with the experimental results. Parametric analysis results recommended that a lower solidification temperature of powder and a higher pre-heated substrate temperature lead to significant reduction of residual stress in $\mathrm{TBC}$.

\section{References}

(1) Arai, M., Sakuma, T., Iwata, U., Saitoh, M., Crack Initiation of Thermal Barrier Coating Layer under Thermal Shock, Journal of the Society of Materials Science Japan Vol. 50 No.3 (2001), pp.297-302.

(2) Arai, M., Sakuma, T., Iwata, U., Saitoh, M., Proposal of Delamination Prediction Method of Thermal Barrier Coating Layer under Thermal Cycle Condition, Journal of the Society of Materials Science Japan Vol. 50 No.6 (2001), pp.651-656.

(3) Matejicek, J., Sampath, S., Brand, P. C., Prask, H. J., Quencing, Thermal and Residual Stress in Plasma Sprayed Deposits: NiCrAlY and YSZ Coatings, Acta Mater. Vol. 47 No.2 (1999), pp.607-617.

(4) Teixeira, V., Andritschky, M., Fischer, W., Buchkremer, H. P., Stover, D., Effects of Deposition Temperature and Thermal Cycling on Residual Stress State in Zirconia-based Thermal Barrier Coatings, Surface and Coaings Technology Vol. 120-121 (1999), pp.103-111.

(5) Ng, H. W., Gam, Z., A Finite Element Analysis Technique for Predicting As-sprayed Residual Stresses Generated by The Plasma Spray Coating Process, Finite Elements in Analysis and Design Vol.41 (2005), pp.1235-1254.

(6) Arai, M., Wada, E., Kishimoto, K., Residual Stress in Thermal Barrier Coating, 2005 Asian Congress on Gas Turbine (stored into CD), Korea

(7) Fujisawa, R., Matsusue, K., Kitahara, K., A Tensile Strength of Structural Ceramics, Journal of the Society of Materials Science Japan Vol. 35 (1986), pp.1112-1117.

(8) Arai, M., Sakuma, T., Mizutani, T., Kishimoto, K., Saitoh, M., Temperature Dependence of Bending Strength for Plasma Sprayed Zirconia Coating, Journal of the Ceramic Society of Japan, Vol. 106 No.2 (1998), pp.198-202.

(9) Kuroda, S., Clyne, T. W., The Quenching Stress in Thermally Sprayed Coatings, Thin Solid Films Vol. 200 (1991), pp.49-66. 